\title{
Comprendre les effets du rapport au droit contenu dans le curriculum caché sur l'éducation à la citoyenneté
}

\section{Understanding the effects of legal consciousness developed through the hidden curriculum on citizenship education}

\author{
Stéphanie Demers ${ }^{1}$, Laurent Paradis-Charette ${ }^{2}$, David Lefrançois ${ }^{3}$, Marc-André Éthier ${ }^{4}$, \\ Maryse Potvin ${ }^{5}$
}

1 Département des sciences de l'éducation, Université du Québec en Outaouais, stephanie.demers@uqo.ca
2 Professionnel de recherche, Université du Québec à Montréal, I.p.charette@gmail.com
${ }^{3}$ Département des sciences de l'éducation, Université du Québec en Outaouais, david.lefrançois@uqo.ca
${ }^{4}$ Département de didactique, Université de Montréal, marc.andre.ethier@umontreal.ca
${ }^{5}$ Département d'éducation et formation spécialisées, Université du Québec à Montréal, potvin.maryse@uqam.ca

RÉSUMÉ. Cet article explore les liens qui s'articulent entre le rapport que les élèves développent aux règles scolaires comme cadre proto-légal faisant partie du curriculum caché et la formation citoyenne. La robustesse du concept de rapport au droit (legal consciouness), défini selon le modèle de Ewick et Silbey (1998), ainsi que la typologie de citoyenneté de Westheimer et Kahne (2004) pour décrire ces liens est évaluée dans une étude de deux cas où les élèves sont appelés à résoudre une situation relative au droit. Les résultats de l'analyse portent à conclure que le dernier niveau du modèle de Ewick et Silbey ne permet pas de distinguer entre une contestation légitime des lois (ou des règles) et un rejet anomique de la loi et qu'il importe de distinguer le droit des droits.

ABSTRACT. This article explores the relationship between how students conceive school rules and codes as a protolegal framework of the hidden curriculum and citizenship education. The robustness of Ewick and Silbey's legal consciouness model, as well as Westheimer and Kahne's citizenship typology in describing this relationship is evaluated through the study of two cases where high school students must resolve a legal situation. The results of the analysis lead to conclude that the third level of Ewick and Silbey's model does not allow for a distinction between legitimately contesting a law and an anomic rejection of the law. It further highlights the importance of distinguishing legal education from rights education.

MOTS-CLÉS. éducation à la citoyenneté, éducation au droit, socialisation politique, école secondaire, rapport au droit, curriculum caché.

KEYWORDS. citizenship education, legal education, political socialisation, high school, legal consciousness, hidden curriculum.

\section{Introduction}

En 2016, Henry Giroux, professeur de l'Université McMaster, signait un texte intitulé « The United States' War on Youth: From Schools to Debtors' Prisons ». Il y faisait notamment état de la judiciarisation croissante des jeunes pour des contraventions aux codes de conduite des écoles et d'une perception publique d'une incivilité et même d'un taux de délinquance croissants chez les jeunes. À cette perception de sens commun s'ajoutent des appels répétés aux autorités publiques pour contrôler une jeunesse qui causerait des problèmes et serait menaçante, en particulier lorsque celle-ci est racisée, appauvrie ou autrement minorisée sociopolitiquement. Au Canada, le gouvernement fédéral a réagi à (et conforté) des perceptions du même genre en apportant neuf amendements à la Loi sur le système de justice pénale pour les adolescents, visant entre autres à «[...] modifier certaines dispositions de la Loi sur le système de justice pénale pour les adolescents (LSJPA) afin de souligner l'importance de la

\footnotetext{
${ }^{1}$ La préparation de cet article et la recherche en cours sont financées par les fonds de recherche de l'équipe Accès au Droit et à la Justice (ADAJ), un partenariat de recherche subventionné par le Conseil de recherches en sciences humaines du Canada (CRSH). 
protection de la société et de faciliter la détention des adolescents récidivistes ou encore qui présentent un danger pour la sécurité publique» (Bibliothèque du Parlement, 2012).

Or, selon Statistiques Canada, «le taux de criminalité chez les jeunes a diminué de $42 \%$ depuis 2000 , ce qui représente un recul plus marqué que la baisse de $34 \%$ du taux global de la criminalité » (Statistique Canada, 2016). Les autorités policières et de justice aux États-Unis établissent le même constat : la criminalité, la délinquance et les effractions commises par les jeunes sont en net recul. Sans même prendre la peine de s'interroger sur la définition des comportements défendus, il serait ainsi possible de postuler que le discours hégémonique sur les jeunes est erroné et qu'il sert un intérêt autre que celui de la paix publique. Néanmoins, il atteint plusieurs sphères de la vie des jeunes et régule l'organisation de leurs rapports sociaux et avec les institutions au quotidien (Brisman, 2012; Stevens, 2013), contribuant ainsi au développement d'un certain rapport au droit étroitement lié à la socialisation politique et l'éducation à la citoyenneté.

Cet article propose d'explorer les écrits qui examinent le rôle de l'École dans la socialisation politique, en particulier en ce qui concerne les différents rapports au droit entretenus par les jeunes. Bien que certaines recherches se soient penchées sur la forme de citoyenneté implicitement ancrée dans les codes de conduites scolaires, c'est-à-dire sur un curriculum caché de formation citoyenne (Raby, $2005 ; 2008$ ), peu de recherches ont examiné ces liens selon des typologies qui permettent de qualifier les actions du citoyen dans ses interactions avec les institutions publiques. À l'inverse, à l'exception de la recherche de Surprenant (2002) sur la socialisation politique et la conscience des droits et devoirs du citoyen dans le cadre d'un programme d'éducation à la citoyenneté, rares sont les recherches qui incluent le rapport au droit dans leur analyse de l'éducation à la citoyenneté formelle et implicite. Afin d'avancer vers une telle recherche, il nous parait essentiel d'identifier un cadre conceptuel permettant d'examiner les rapports au droit formulés de façon implicite dans les cadres normatifs scolaires et le type de citoyen formé dans un tel cadre. Ce sera le but de cet article.

En premier lieu, nous aborderons le mandat confié à l'école secondaire dans cette socialisation, notamment en fonction du discours public sur les jeunes. En deuxième lieu, nous présenterons deux typologies - du rapport au droit et de l'éducation à la citoyenneté - outillant l'analyse critique des liens qu'entretiennent une typologie des rapports au droit (Ewick et Silbey, 1998) avec les visées de l'éducation à la citoyenneté en contexte scolaire (Westheimer et Kahne, 2004). Enfin, deux cas de figure seront soumis à l'analyse afin de tester cette typologie et d'examiner l'importance de la prise en compte du rapport au droit (aux règles et aux normes) dans la formation citoyenne des jeunes.

\section{Problématique}

Les jeunes seraient en effet assujettis à un important dispositif de régulation et de contrôle social, notamment en raison d'une perception, chez certains adultes, qu'ils représentent une menace pour l'ordre, en particulier lorsqu'il s'agit de jeunes racisés ou autrement minorisés (Brisman, 2012; Giroux, 2000, 2009; Hirshfield, 2008; Eid, Magloire et Turenne, 2011), voire que la jeunesse constitue un problème en soi, qu'il importe de régler. La médiatisation d'actes criminels par des jeunes, de leur taux de chômage ou de décrochage de l'école tend à perpétuer une conception de la jeunesse incontrôlable, à subjuguer entre autres par les réformes législatives et sociales « Proposed solutions are driven by the idea of youths as unruly bodies in need of external containment » (Adelman et Yalda, 2000, p. 37). La résistance dont font preuve certains jeunes aux différentes formes de contrôle social serait rejetée, voire criminalisée; elle alimenterait les appels de certains adultes à un renforcement de l'autorité physique et morale de l'école sur les élèves, la revalorisation des «valeurs traditionnelles 》 et l'accroissement du rôle « parental» de l'État par le biais de ses institutions, notamment scolaires et juridiques (Adelman et Yalda, 2000; Giroux, 2009). Le concept de «jeune » serait souvent considéré comme synonyme de dangerosité, dangerosité qu'il convient de contrôler et de subordonner à l'autorité de l'école; les jeunes issus des classes populaires éprouvant des difficultés à l'école sont d'autant plus ciblés qu'ils sont exclus de leur fonction sociale par le jugement scolaire les concernant. Par extension, « la scolarité obligatoire a pour effet de définir les jeunes comme appartenant à une sous-catégorie et d'imposer à 
leurs mouvements et à leurs perceptions de certains types de restrictions auxquelles personne d'autre n'est soumis » (Friedenberg, 1969; cité par Lemay, 2000, p. 89-90). Prost (1997) renchérit et rappelle que l'école, chargée de cette surveillance,

«[...] ne peut rien imaginer qui lui permette de donner aux élèves les plus grands [adolescents] une part de responsabilité et d'initiative. Mais l'école n'est pas seulement contraignante par son comportement quotidien: elle l'est aussi en elle-même, par sa signification institutionnelle, puisque c'est le moyen inventé par les adultes pour retarder l'entrée des jeunes dans la vie active et se défendre contre la menace latente qu'ils font peser sur les ordres établis.» (p. 483)

Des règlements municipaux qui interdisent le regroupement des jeunes dans les parcs ou qui imposent un couvre-feu, par exemple, jusqu'à l'imposition grandissante de l'uniforme scolaire, sensé aplanir les symboles d'appartenance sociale jugés potentiellement dangereux ou sources de conflits, les jeunes seraient une cible privilégiée des institutions de contrôle social (Hirshfield, 2008; Raby, 2008). Les conséquences leur seraient préjudiciables, dans la mesure où plusieurs sites de leur vie quotidienne, comme l'école ou les parcs municipaux, présenteraient des contextes d'attentes réduites de respect de leurs droits et libertés fondamentales. À l'instar d'Adelman et Yalda (2000), de Brisman (2012) et Stevens (2013), nous postulons que le rapport que les jeunes entretiennent à la loi, au droit et à la justice de façon générale conditionne l'accès au droit et à la justice et donc le développement de leur citoyenneté, que le droit est « un outil de définition et de nomenclature des rapports sociaux [et qu']il est aussi un médium de conditionnement et de construction sociale » (Surprenant, 2002, p. 1). Les individus constitueraient ce rapport par leurs représentations sociales et leurs savoirs au sujet du droit et de la justice, leurs expériences impliquant les concepts de droits et de justice, ainsi que les attitudes et attentes qu'ils développent en interaction avec eux (Silbey, 2005). La vie quotidienne des jeunes serait notamment façonnée par leurs interactions avec les adultes dans des cadres régis par la loi et le droit, les cadres scolaire, familial, du travail et communautaire, entre autres. Ces cadres habiliteraient ou contraindraient l'action des jeunes et contribueraient à un processus de socialisation juridique et politique qui inclut le type de rapport que les jeunes développent avec la loi, le droit et les droits comme dimension fondamentale de leur conscience citoyenne (Raby, 2005). Silbey (2005) souligne de plus que le rapport au droit peut agir sur la définition qu'ont les individus de leur pouvoir et de leur agentivité citoyenne, ainsi que du pouvoir et de l'autorité des institutions politiques, législatives et exécutives. Il habiliterait ou contraindrait, selon sa nature, l'action citoyenne au sein ou contre ces institutions. Des recherches empiriques tendent à indiquer que la construction des perceptions que les personnes ont de la loi, de l'autorité et des institutions légales, comme composantes essentielles de leur citoyenneté, commence à l'enfance (Chriss, 2007). Bien que ces orientations initiales évoluent dans le temps, l'adolescence constituerait un moment charnière pour le développement des croyances politiques et sociales, les perceptions à l'égard de la justice formées à l'adolescence persistant dans la vie adulte (Hagan, Shedd et Payne, 2005; Carr, Napolitano et Keating, 2007; Stevens, 2013; Surprenant, 2002). Le processus de socialisation associé à l'émergence d'une conscience du droit dépasse toutefois le seul cadre de la loi et du droit. En effet, tel qu'avancé par Adelman et Yalda (2000), ainsi que par Brisman (2012) et Stevens (2013), les institutions comme l'école contribueraient à une conception du contrôle social formel et de l'autorité institutionnelle qui étend le rapport au légal et au droit aux règles et normes codifiées pour réguler la vie quotidienne des jeunes. Certaines recherches (Raby, 2008; Stevens, 2013) permettent effectivement de constater que les rapports que les élèves établissent aux règles scolaires seraient liés de manière étroite aux rapports qu'ils établissent au droit, aux droits et à la justice, ainsi qu'à leur conceptions de l'organisation des rapports sociaux.

L'éducation à la citoyenneté serait ainsi autant le produit de diverses prescriptions curriculaires formelles encadrant l'éducation à la citoyenneté à l'école que du cumul des expériences quotidienne des élèves dans les interactions quotidiennes avec leurs pairs, enseignants, directions, personnel de soutien, c'est-à-dire dans ces rapports sociaux encadrés par les règles scolaires et les «droits » des élèves, qui caractérisent le curriculum caché. Implicite et manifeste dans les formes non discursives de 
l'école, c'est-à-dire dans les expériences pratiques dont le message imprègne le silence structuré, le curriculum caché réifierait l'ordre scolaire et la légitimité du pouvoir de l'autorité scolaire comme institution «législative» (Demers, 2011; Giroux, 1981). Giroux (1981) rappelle qu'un curriculum scolaire est entendu comme ensemble de prescriptions explicites, planifiées et consciemment formulées et appliquées en fonction de visées éducatives socialement consensuelles. Le curriculum caché, pour sa part, n'est pas planifié et reste informel, dans les pratiques et interactions routinières des acteurs scolaires (habituellement à leur insu) comme porteur d'hégémonie en termes de normes légitimes dans les interactions sociales. Il importe toutefois de noter que le concept de curriculum caché se distingue selon qu'il est abordé dans une perspective structurale fonctionnaliste, comme le font Durkheim (1934), Jackson (1968) et Drebeen (1968), dans une perspective phénoménologique ou encore selon une analyse critique néomarxienne (Giroux et Penna, 1979). Sans le nommer ainsi, Durkheim (1934/2008) décrit le curriculum caché dans cette même visée de formation citoyenne, comme «discipline du corps social» (p. 105), constitué d'un système de règles ...

«[...] qui prédéterminent la conduite de l'enfant. Il doit venir en classe avec régularité, il doit s'y présenter à heure fixe, dans une tenue et une attitude convenables; en classe, il ne doit pas troubler l'ordre ; il doit avoir appris ses leçons, fait ses devoirs, et les avoir faits avec une suffisante application, etc. Il y a ainsi une multitude d'obligations auxquelles l'enfant est tenu de se soumettre.» (p. 105)

dont la fonction sociale est d'imprégner l'enfant d'une morale du devoir de l'élève, qui deviendra celui du citoyen face à l'État et du travailleur face à l'employeur. Cette morale scolaire sert

«d'intermédiaire entre la morale affectueuse de la famille et la morale plus sévère de la vie civile. C'est en respectant la règle scolaire que l'enfant apprendra à respecter les règles, qu'il prendra l'habitude de se contenir et de se gêner, parce qu'il doit se gêner et se contenir. C'est une première initiation à l'austérité du devoir.» (p.106)

c'est-à-dire qu'elle agit comme formation civique ou citoyenne. Jackson (1968) sera le premier à définir cet ensemble de valeurs et d'attentes normatives comportementales, sociales et dispositionnelles comme curriculum caché, situé au carrefour du curriculum formel, structuré autour de savoirs scolaires, et de la régulation sociale au sens large, structurée autour d'obligations sociales et politiques. Selon Drebeen (1968) ce processus de socialisation par l'École qu'est le curriculum caché préparerait les élèves à participer aux rapports intersubjectifs et collectifs qui caractérisent la sphère publique et du travail, notamment en subsumant les différences issues du contexte familiale ou socioculturel, voire même de l'identité singulière. La structure scolaire, en ce sens, conditionnerait un rapport de soumission à l'autorité et une conception du traitement différencié et catégoriel comme légitime (Drebeen, 1968). Giroux et Penna (1979) soulignent que cette perspective du curriculum caché n'aborde pas les conflits inhérents à la divergence d'intérêts (notamment socioéconomiques) entre groupes sociaux, ne problématise pas les valeurs, normes et attentes véhiculées par l'École et définit les élèves comme produits passifs du processus de socialisation. À l'inverse, la perspective phénoménologique (O’Neill, 1973; Young, 1976) conçoit le curriculum caché comme co-construit par tous les acteurs scolaires dans le sens qu'ils donnent à leurs interactions. Elle questionne en ce sens la distribution et la légitimité du pouvoir de ces acteurs dans le contexte scolaire. Selon Giroux et Penna (1979), la faiblesse principale de cette perspective réside dans son idéalisme social qui néglige l'asymétrie des rapports de pouvoir dans l'École et le rôle de l'idéologie dominante dans les représentations qu'ont les élèves du monde social. Dans une perspective critique, enfin, Bowles et Gintis (1976), Giroux et Penna (1979) définissent le curriculum caché dans son rapport à la reproduction culturelle et économique. Comme agent de contrôle idéologique, l'École reproduirait par les pratiques légitimées de ses acteurs les croyances, valeurs et normes dominantes (lesquelles constituent le curriculum caché) et conséquemment, les relations sociales qui caractérisent les structures hégémoniques : rapports de production, injustices économiques, illusions de pouvoir et fausse conscientisation (Bernstein, 1977). Apple (2004) avance dans le même sens que le curriculum caché a comme fonction de maintenir le pouvoir de la culture dominante et les relations de classes 
existantes et qu'il entre ainsi en contradiction avec les énoncés curriculaires formels quant à la formation citoyenne, notamment par la pacification des conflits inhérents à l'interaction intersubjective et à l'exercice de droits.

Cette contradiction est soulevée dans la recherche de Surprenant (2002) et les recherches de Raby $(2005 ; 2008)$. «Alors que l'éducation institutionnalisée offre aux citoyens la possibilité de contribuer au développement de sa communauté de façon consciente et éclairée par le biais de ses diverses occupations, elle ne fournit pas le suivi et le support nécessaire à leur participation pleine et entière dans les rapports "humains". » (Surprenant, 2002, p. 10). En d'autres mots, les citoyens en formation que sont les élèves sont souvent ignorants de leurs droits civils, politiques, sociaux et culturels et peu encouragés à leur exercer (Raby, 2008), vidant ainsi le sens même d'une citoyenneté "pleine et entière ». De plus, le rapport au droit et à la justice enchâssé dans le curriculum caché - dont les règles de vie scolaire - conditionnerait à une forme particulière de citoyenneté, soumise aux institutions et aux rapports de domination qui caractérisent l'économie capitaliste (Raby, 2005).

L'élève ingouvernable, dans une telle perspective, c'est-à-dire celui qui ne se conforme pas aux règles de gouvernance capitaliste, est, ou devient, Autre, objet de contrôle et de répression. Les résultats de l'enquête menée par Eid, Magloire et Turenne sur le profilage racial et la discrimination auprès de jeunes racisés au Québec (2011) font état d'un traitement scolaire différencié et discriminatoire pour ces élèves, souvent porté par une surveillance accrue de leurs activités et un recours abusif aux règles scolaires pour appliquer des sanctions plus sévères que celles qui seraient appliquées aux comportements d'élèves non racisés. Ces résultats convergent avec ceux obtenus par Morrill et ses collègues (2010) dans leur recherche auprès de plus de 5000 élèves racisés et non-racisés et leurs expériences de violations de leurs droits par l'institution scolaire. Comme l'ont avancé Bowles et Gintis (1976), Foucault (1977) et plus récemment Wacquant (2001) et Giroux (2003), « une discipline stricte dans les écoles de la classe ouvrière aide à promouvoir des transitions harmonieuses et volontaires vers un monde de travail industriel qui régule et subordonne les travailleurs de façon étroite. » (Hirschfield, 2008, p. 80). Les résultats de Morrill et ses collègues portent en effet à croire que, même lorsqu'ils sont conscients des atteintes à leurs droits, les élèves ne font pas appel au droit ou à la loi pour confronter la situation et considèrent qu'ils n'ont pas le pouvoir d'agir contre l'adulte à l'origine de la situation: moins de $5 \%$ des élèves ayant indiqué avoir vécu une telle violation ont eu recours au droit, à une action légale quelconque (Morrill et al., 2010). Le fait d'être jeune face aux adultes et Autre (minorisé) face aux institutions «majoritaires » contribuerait à une conscience légale - une conception de sens commun quant au fonctionnement du droit et de la loi — selon laquelle ce fonctionnement est défaillant au sein de l'école et que les élèves n'ont en pratique que peu de pouvoir d'agir sur leurs expériences quotidiennes et rapports sociaux qui les caractérisent.

Or, si les règles scolaires et l'organisation institutionnelle des rapports sociaux quotidiens des jeunes contribuent de façon importante à former leur conscience du droit et de la justice (Fagan et Tyler, 2005; Raby, 2008), il semble opportun de questionner quelle conception de la citoyenneté en découle et en quoi cette dernière est cohérente ou non avec les visées de formation citoyenne de l'école telles qu'énoncées dans le curriculum formel. Plusieurs situations récentes de résistance d'élèves à de telles attentes fournissent des cas de figure intéressants de leur construction en Autres à discipliner ou à marginaliser. Nous proposons d'analyser deux de ces cas, en mobilisant le cadre conceptuel qui suit.

\section{Cadre conceptuel}

\subsection{Le rapport au droit d'Ewick et Silbey (1998)}

Le concept de conscience du droit tire son origine des études critiques du droit des années 1970, qui conçoit ces dernières comme un système de domination, dans une analyse culturaliste praxéologique (Pélisse, 2005) qui mobilise les concepts de conscience, d'idéologie et d'hégémonie afin d'expliquer comment un système légal peut produire d'importantes inégalités tout en étant toléré. Dans les années 1980, Silbey \& Bittner identifient la conscience du droit comme étant l'élément clé permettant 
de comprendre l'écart entre le droit tel qu'écrit et le droit dans son usage, vécu dans les rapports sociaux, qui incluent l'exercice de la citoyenneté (1982, p. 399). La conscience du droit permettrait de comprendre la matérialisation de cette tolérance dans le contexte particulier du droit comme pratique culturelle (Merry, 1985). Dans cette mouvance des Legal Counsciousness Studies, qui portent leur regard sur l'expérience concrète qu'ont des acteurs sociaux du système légal plutôt qu'au système légal lui-même, Silbey (1982), Sarat (1990) et Brigham s'intéresseront respectivement au droit comme pratique sociale, au droit en action et à l'impact de la compréhension du droit sur l'organisation des rapports sociaux (Pélisse, 2005). D'autres modèles ont été proposés pour expliquer le rapport que les individus entretiennent au droit, dont le modèle culturel de Kourilsky (1991) qui situe la socialisation juridique dans l'imprégnation culturelle et le modèle cognitiviste en psychologie (Tapp et Kohlberg, 1971; Jakubowska, 1991). Inspirés notamment de la théorie du développement moral de Kohlberg (1976), ce dernier a une perspective plus étroite, individualisante du phénomène, réduisant l'interaction entre l'individu seul et le droit, tenant en conséquence peu compte de sa dimension sociale. À l'instar des sociologues et anthropologues du droit cités plus haut, toutefois, nous considérons que tout rapport individuel au droit est d'abord le produit d'interactions et de constructions sociales.

Ewick et Silbey (1998) s'intéressent dès les années 1990 aux usages sociaux des individus qui en viennent à interpréter et rendre signifiant le droit (Pélisse, 2005). Leur approche comporte un entretien en trois phases: les chercheurs demandent aux participants de décrire leur environnement social de proximité et de leur vie quotidienne; puis ils leur demandent de décrire les évènements et pratiques qui les auraient dérangés ou troublés; enfin, ils se concentrent sur un de ces évènements particuliers choisis par le participant rencontré. Par cette méthodologie, les chercheures identifient trois manières de se rapporter au droit et de construire la légalité soit face au droit (Before the law), avec le droit (With the law) et contre le droit (Against the law). Cette typologie est particulièrement intéressante, car elle peut s'appliquer aux divers ensembles de normes et codes qui régissent la vie quotidienne des individus et des groupes, dans leurs interactions et avec les institutions publiques, (re)considérant l'État et le rôle de l'autorité publique (Pélisse, 2005). Elle contient conséquemment une conception implicite de la citoyenneté, bien qu'elle ne soit pas mobilisée en ce sens.

En premier lieu, l'individu face au droit concevrait la légalité, les lois comme existant dans une sphère séparée de la vie quotidienne, immuable et transcendant les conflits banals et les histoires humaines qui marquent la vie ordinaire. Pour cet individu, le droit est un système rationnel, formellement ordonné et hors de son contrôle. En deuxième lieu, l'individu qui conçoit un rapport avec le droit y voit des règles et procédures lui préexistant, mais dont il peut se servir pour faire avancer ou défendre ses intérêts. Ainsi, les compétences et ressources des acteurs qui agissent avec le droit, entre autres dans l'interprétation qu'ils en font, dans les négociations autour de leur interprétation et dans l'invention, par la jurisprudence, par exemple, de nouvelles règles du jeu légal, leur permettent d'agir légitimement dans leur intérêt. Enfin, l'individu qui entretient un rapport contre le droit se concevrait comme pris par le cadre légal comme pouvoir arbitraire, contre lequel il se sentirait impuissant (Pélisse, 2005 p.123-124). Chacun de ces types de conscience du droit possède une normativité, un type de contraintes, une capacité, un espace-temps et un archétype particulier que Pélisse (2005) simplifie dans le tableau suivant : 


\begin{tabular}{|c|c|c|c|}
\hline & Before the law & With the law & Against the law \\
\hline Normativité & $\begin{array}{l}\text { Impartialité, } \\
\text { objectivité de la loi et } \\
\text { du droit }\end{array}$ & $\begin{array}{l}\text { Légitimité partielle du } \\
\text { droit, recherche de son } \\
\text { propre intérêt }\end{array}$ & $\begin{array}{l}\text { Pouvoir du droit, « la } \\
\text { force fait le droit » } \\
\text { (might makes right) }\end{array}$ \\
\hline Contraintes & $\begin{array}{l}\text { Liées aux structures } \\
\text { organisationnelles }\end{array}$ & $\begin{array}{l}\text { Contingentes, closes } \\
\text { (par une décision, un } \\
\text { verdict, des } \\
\text { compétences et } \\
\text { ressources) }\end{array}$ & $\begin{array}{l}\text { Visibles } \\
\text { institutionnellement }\end{array}$ \\
\hline Capacité & $\begin{array}{l}\text { Donnée par les règles, } \\
\text { l'organisation formelle }\end{array}$ & $\begin{array}{l}\text { Liée à des ressources } \\
\text { individuelles, } \\
\text { expériences, habileté }\end{array}$ & $\begin{array}{l}\text { Insérée dans les } \\
\text { structures sociales } \\
\text { (rôles, règles, } \\
\text { hiérarchie) }\end{array}$ \\
\hline Temps/Espace & $\begin{array}{l}\text { Séparé de la vie de } \\
\text { tous les jours }\end{array}$ & $\begin{array}{l}\text { Simultané à la vie de } \\
\text { tous les jours }\end{array}$ & $\begin{array}{l}\text { Colonisant le temps/ } \\
\text { l'espace de la vie de } \\
\text { tous les jours }\end{array}$ \\
\hline Archétype & Bureaucratie & Jeu & Débrouillardise \\
\hline
\end{tabular}

Tableau 1. Rapports au droit selon la typologie de Ewick et Silbey (1998) (adapté de Pélisse, 2005)

\subsection{Le rapport aux règles et codes de conduite scolaires comme extension du rapport au droit}

Les résultats recherche de Raby (2008) sur les rapports que les élèves entretiennent avec les règles et les codes de conduite scolaires illustrent que ces derniers peuvent être caractérisés de façon analogue à la typologie des rapports au droit de Ewick et Silbey (1998), dans la mesure où des élèves se concevraient comme étant passifs face aux règles scolaires immuables, voire transcendantes, d'autres se situeraient contre les règles, dans la mesure où elles seraient transgressables et, enfin, certains conçoivent un rapport avec les règles, impliquant qu'ils peuvent en négocier les interprétations dans leurs intérêts. Les codes de conduite examinés par Raby (2005) contiennent une dimension associée à la conscience du droit et à la citoyenneté, notamment dans leur traitement des droits et responsabilités de l'élève, et contribueraient en ce sens à créer un habitus qui applique et renforce les valeurs de la bourgeoisie. La citoyenneté qui en découlerait serait caractérisée par la retenue, la docilité et un fort ancrage au sein d'une hiérarchie, des relations de propriété privée, de genre et d'âge, entre autres (p. 75). À ce titre, Raby (2005) avance que les règles scolaires, les codes de conduites et les uniformes sont des sites par lesquels les subjectivités (par exemple, le « citoyen responsable ») sont créées et les modes de gouvernance intériorisés sont alimentés pour produire des élèves - des adultes citoyens en devenir - conformes aux cadres capitalistes. À l'école, l'injonction à l'autogouverne de type néolibéral agirait conjointement avec l'imposition «top-down » du pouvoir disciplinaire et la punition ou la sanction, qui distingue le «bon» citoyen du « mauvais », ce qui n'est pas sans rappeler les tensions entre l'individu et les institutions publiques dans les structures sociopolitiques en général (Lessard, 2008), où la définition du « bon » est liée de façon inhérente aux valeurs d'une classe sociale dominante. Ce paradoxe est d'autant plus puissant dans un contexte où les sujets à qui l'on demande de s'autogouverner sont assaillis de part et d'autre par une conception de l'adolescence comme étape d'irrationalité, d'être en devenir qui requiert l'intervention disciplinaire des figures d'autorité.

Raby (2005) souligne que la conception des droits des élèves dans le milieu scolaire est une conception négative, c'est-à-dire associée au droit à être protégé contre quelque chose, rarement au droit de, c'est-à-dire selon une conception capacitante, autonomisante ou émancipatrice: « Almost all codes under study instead emphasized passive citizenship through a concentration on protective rights (such as the right to be in a safe environment and to education). » (p. 76) Plus encore, des codes de 
conduite scolaires présenteraient les droits comme contingents, et en conséquence précaires, puisqu'ils dépendraient du fait que les élèves remplissent ou non des responsabilités. Une citoyenneté découlant d'une telle conception des droits serait méritée plutôt qu'inhérente à la condition d'élève. Ainsi, aucun code de conduite n'enchâsse le droit à la libre expression, d'être entendu, de présenter ses doléances ou son désaccord avec un règlement ou une politique scolaire. Dans les cas où les élèves sont consultés, ils ne disposent pas de droit de vote leur reconnaissant un rôle d'agent actif dans la vie de l'école et l'exercice de droits. À l'instar de Devine Devine (2002), Raby (2005) postule que la citoyenneté développée dans un tel contexte est une manifestation de la gouvernance néolibérale qui exige l'autorégulation et l'autogestion d'un citoyen responsable et prudent, parce que responsable et redevable de ses propres risques. L'agentivité de l'élève dans un tel cadre se limite à son autodiscipline.

Dans ses analyses, Raby décrit la citoyenneté découlant des codes de conduite en fonction du rapport des élèves au pouvoir et à l'autorité (docilité, obéissance), mais également selon la nature des droits (passifs et négatifs) que leur reconnait l'institution à travers ses codes et ses normes. Cette analyse fait peu état de l'agentivité des élèves en lien avec ces règles ou les horizons d'actions possibles qui résultent de leur intériorisation. Cette limite au cadre conceptuel nous invite à chercher une autre façon de concevoir la citoyenneté qui émerge du rapport que les élèves entretiennent aux codes et aux normes de l'école comme curriculum caché.

\subsection{Les visées de l'éducation à la citoyenneté}

Lefrançois (2006), Pagé (2001) et Weinstock (2000), notamment, ont décrit de façon synoptique et analysé l'expression sociopolitique et le développement historique de divers modèles normatifs de démocratie et de citoyenneté, du républicanisme aristotélicien ou rousseauiste (priorité de la volonté populaire [ou de la liberté des Anciens] sur les droits individuels [ou la liberté des Modernes]) à la théorie politique délibérative habermassienne (caractère cooriginaire de la volonté populaire et des droits individuels), en passant par le libéralisme lockien (priorité des droits individuels sur la volonté populaire) qui se poursuit dans les travaux majeurs de philosophes politiques comme Rawls ou Dworkin. Cela dit, dans cet article, nous postulons plutôt, à l'instar de la théorie critique de type marxiste, que la démocratie et la dictature représentent deux formes corrélées de pouvoir, lequel peut s'étendre aux plans économique, politique, social, etc. En régime capitaliste, la démocratie politique est souvent un paravent qui permet aux propriétaires de moyens de production de ce territoire d'agir dans leur intérêt collectif à long terme (notamment en luttant contre d'autres États pour la répartition de la plus-value soutirée aux exploités, en développant les infrastructures et les superstructures, en divisant, domestiquant ou réprimant les exploités et les gouvernés) et de maintenir ainsi leur domination et leurs privilèges culturels, économiques et sociaux, voire d'optimiser les ressources humaines et naturelles et de les exploiter à leur guise (Ethier, 2003). Comme le concept de démocratie ou de citoyenneté luimême, l'éducation à la citoyenneté a fait l'objet de nombreux débats politiques et scientifiques. Cependant, dans le cadre restreint de ce texte, ne fournissons que quelques exemples. Cette éducation à la citoyenneté doit-elle amener l'élève à adhérer à une idéologie et à adopter des comportements socialement acceptables (refuser la violence, voter, payer ses impôts, respecter l'autorité, etc.) ou à se désaliéner en apprenant des attitudes, des opérations de pensée et des valeurs permettant de se poser des questions de fond sur la société, d'y répondre avec méthode et rigueur, de délibérer et d'agir avec conséquence? Vise-t-elle l'intégration individuelle à l'élite ou la solidarité collective avec les opprimés, l'identité ou la critique, le consensus ou la contestation, la conservation des traditions ou le changement social radical ? (Ethier, 2001). Quoi qu'il en soit, l'éducation à la citoyenneté « fait entrer en jeu aussi bien des connaissances et [des] savoirs que des valeurs ou encore des comportements. Ses finalités sont ambitieuses et les injonctions qui lui sont faites souvent contradictoires » (Audigier, 2007, p. 25).

Lefrançois (2007) rappelle toutefois que l'éducation à la citoyenneté dans sa proposition scolaire dominante 
« [...] implique la transmission de connaissances qui servent de base à l'organisation étatique du droit et de la démocratie dans le Québec contemporain (Ministère de l'Éducation, 2004, p. 348-349). Il s'agit de présenter les institutions juridiques et les libertés conférées aux citoyens, de développer des aptitudes politiques participatives et de construire une nouvelle identité sociale partagée en contexte de pluralisme. Ces finalités de la formation civique coïncident avec les dimensions (juridique, politicoparticipative et socioidentitaire) de la citoyenneté $[\ldots] »$ (p. 5).

Ainsi, dans sa forme actuelle, l'éducation à la citoyenneté pourrait être conçue selon plusieurs perspectives qui recoupent des finalités et des approches éducatives diverses. Scott-Baumann (2003) définit les fondements de l'éducation à la citoyenneté dans les curricula comme développement de la littératie politique « fondée sur les lois encadrant les droits de la personne et une vision humaniste de la responsabilité »(p. 355). Le cadre politico-légal formerait la base de la citoyenneté comme débat public rationnel. Ce cadre serait également au centre de la définition de l'éducation civique formulée par Feldman (2007) en général « définie comme étude des gouvernements local, régional et fédéral et des droits et responsabilité des citoyens » (p. 4). Pour sa part, Print (2007) avance que l'éducation à la citoyenneté est un programme formel qui fournit l'occasion d'apprendre en quoi consiste le système de gouvernement de sa société, la démocratie, la société de droits, les valeurs démocratiques, les savoirs et les compétences associées à l'analyse, la prise de position et l'action face aux enjeux politiques.

Dans leurs travaux, Westheimer et Kahne (2004; Khane et Westheimer 2006) définissent la citoyenneté comme la conception du coffre à outils sociaux idéal pour permettre aux citoyens de s'épanouir et favoriser le développement d'une société démocratique. Dans ce cadre, ils s'intéressent à la variété des «destinations » de citoyenneté existante pour les citoyens. ${ }^{2}$ En raison de l'importance accordée aux actions quotidiennes et routinisées des acteurs sociaux, de la façon dont ces actions témoignent d'une conception du rôle que ses acteurs jouent dans la polis et face au politique, le modèle de Westheimer et Kahne s'approche du fondement culturaliste praxéologiste adopté par Ewick et Silbey dans leur typologie du rapport au droit, favorisant un croisement théorique cohérent.

Dans leur recherche sur les programmes d'éducation à la citoyenneté dispensés dans dix écoles étatsuniennes, Westheimer et Kahne (2004) ont analysé les données issues du croisement de quatre collectes de données distinctes, soit: l'observation dans les classes et dans les sites de services d'organismes de promotion d'un programme éducatif; un corpus d'entrevues (d'élèves, d'enseignants et de responsable de programme); des sondages pré et post exposition au programme. Ils ont ainsi pu dégager une typologie du citoyen formé par ces programmes qui traduisent, par les choix de contenus, d'approches et d'activités d'apprentissage, une conception de ce qu'est le «bon» citoyen. Trois conceptions $\mathrm{du}$ «bon» citoyen émergent de leur recherche. On retrouve d'abord le citoyen personnellement responsable: il entretient une relation individualisée avec l'État; il agit de façon responsable au sein de sa communauté, obéit aux lois, fait don de sang, contribue aux cueillettes pour les démunis et fait du bénévolat, par exemple. Sous-jacente à cette conception de la citoyenneté est l'idée que les problèmes sociaux sont issus d'une déficience individuelle de caractère de certains citoyens et que leur résolution réside dans les gestes des citoyens de bon caractère (honnêtes, responsables, qui obéissent aux lois). On nie par le fait même la possibilité que les problèmes de la société soient structurels. Il s'agirait ici d'un citoyen qui se place face au droit comme en soi immuable et transcendant auquel il doit allégeance et obéissance.

Ce type de citoyenneté est analogue à ce que Crick (2007) appelle la citoyenneté minimaliste: obéir aux lois, payer ses impôts, conduire avec prudence, agir de façon socialement acceptable, sans toutefois agir avec d'autres sur des enjeux qui peuvent influencer les politiques publiques. Il s'agit en somme d'une vision selon laquelle « competitive elections create governments that can modify and uphold a legal order under which individuals can lead their lives with as little interference as possible from the state and minimal public obligations » (Crick, 2007, p. 244). Il n'en demeure pas moins que ce type de citoyen peut aussi être un «bon » citoyen dans une autocratie. 
La seconde conception identifiée par Westheimer et Kahne (2004) est celle du citoyen participatif, qui s'engage à participer dans sa communauté à divers paliers. Par exemple, le citoyen participatif planifie et participe de façon active dans des efforts communautaires pour encourager le recyclage, venir en aide aux démunis, etc. Il s'agit de citoyens qui comprennent le fonctionnement des institutions publiques et des organismes communautaires et y assument un certain leadership. Cette conception du citoyen traduit une vision de la résolution des problèmes sociaux comme émanant de la participation active des citoyens dans les systèmes étatiques et communautaires établis. Elle est cohérente avec la vision libérale de la démocratie comme tributaire d'un haut niveau de participation citoyenne à ses institutions «the maintenance of free institutions depends on a high level of popular participation in public affairs, both as a practical necessity and as a moral and civic duty » (Crick, 2007, p. 244). Il est possible de postuler un rapprochement entre ce type de citoyenneté et l'idée d'être avec le droit, dans la mesure où les citoyens participatifs se servent du droit pour faire avancer leur cause, pour encadrer leur participation et celle des autres, pour négocier des espaces et des ressources dans les intérêts qu'ils défendent.

Le troisième type de citoyen qui émerge de l'étude de Westheimer et Kahne (2004) est un citoyen orienté vers la justice, dont les efforts analytiques et rhétoriques sont déployés en quête de justice sociale. Au contraire des deux autres types, ce citoyen problématise et dénonce les structures qui engendrent l'injustice et ne peut être satisfait des institutions publiques et de l'ordre social tel qu'ils existent. Ce citoyen s'érigerait plutôt contre le droit comme outil d'oppression créé par une élite pour défendre ses propres intérêts.

Enfin, dans leur analyse de cette typologie, Lefrançois, Éthier et Demers (2009) avancent que

«Cette typologie ne comporte toutefois pas de catégorie à part pour les citoyens qui mèneraient une lutte révolutionnaire et renverseraient l'ordre établi, s'il dépendait de l'exploitation et de l'oppression ou s'il en générait. Pourtant, l'on peut penser que l'établissement de la justice serait dans l'intérêt d'une majorité des citoyens et nécessiterait, le cas échéant, leur engagement dans des actions de nature collective visant, par exemple, à changer le mode de propriété des moyens de production et rendre réciproques, à diverses échelles, les rapports de pouvoir et les relations sociales. En définitive, la figure d'un Thomas Sankara ou d'une Rosa Luxembourg est absente. » (p. 75)

Chacun de ces types de citoyens possède une normativité, une intention, une capacité, une idéologie et un archétype particulier que l'on peut résumé dans le tableau suivant: 


\begin{tabular}{|c|c|c|c|c|c|}
\hline & $\begin{array}{c}\text { Citoyen } \\
\text { Aliéné/anomique }\end{array}$ & $\begin{array}{c}\text { Citoyen } \\
\text { responsable }\end{array}$ & $\begin{array}{c}\text { Citoyen } \\
\text { Participatif }\end{array}$ & $\begin{array}{c}\text { Citoyen } \\
\text { orienté vers la } \\
\text { justice }\end{array}$ & $\begin{array}{c}\text { Citoyen } \\
\text { révolutionnaire }\end{array}$ \\
\hline Normativité & Égoïsme & $\begin{array}{l}\text { Respect des } \\
\text { règles }\end{array}$ & $\begin{array}{l}\text { Participation } \\
\text { aux instances }\end{array}$ & $\begin{array}{l}\text { Dénonciation } \\
\text { du pouvoir }\end{array}$ & $\begin{array}{l}\text { Action } \\
\text { révolutionnaire }\end{array}$ \\
\hline Intention & $\begin{array}{c}\text { Profiter du } \\
\text { système et des } \\
\text { autres }\end{array}$ & $\begin{array}{l}\text { Éviter le conflit } \\
\text { (produire des } \\
\text { citoyens } \\
\text { obéissants) }\end{array}$ & $\begin{array}{c}\text { Atténuer le } \\
\text { conflit (Produire } \\
\text { des citoyens qui } \\
\text { agissent à } \\
\text { l'intérieur du } \\
\text { cadre accepté) }\end{array}$ & $\begin{array}{c}\text { Analyser et } \\
\text { dénoncer les } \\
\text { racines } \\
\text { structurales du } \\
\text { conflit } \\
\text { (Produire des } \\
\text { citoyens } \\
\text { critiques) }\end{array}$ & $\begin{array}{c}\text { Produire des } \\
\text { acteurs du } \\
\text { changement } \\
\text { social visant la } \\
\text { réciprocité } \\
\text { humaine } \\
\text { (Transformer la } \\
\text { société) }\end{array}$ \\
\hline Capacité & $\begin{array}{l}\text { Détourner ou } \\
\text { enfreindre les } \\
\text { règles à son profit }\end{array}$ & $\begin{array}{l}\text { Obéissance: } \\
\text { Suivre les } \\
\text { règles et } \\
\text { l'organisation } \\
\text { formelle }\end{array}$ & $\begin{array}{c}\text { Participation: } \\
\text { Implication } \\
\text { individuelle dans } \\
\text { les structures } \\
\text { organisationnelles }\end{array}$ & $\begin{array}{c}\text { Critique } \\
\text { discursive de } \\
\text { la racine des } \\
\text { problèmes } \\
\text { sociaux et } \\
\text { implication } \\
\text { collective }\end{array}$ & $\begin{array}{c}\text { Renverser } \\
\text { l’ordre établi } \\
\text { par des actions } \\
\text { collectives }\end{array}$ \\
\hline « Idéologie » & Pragmatisme & $\begin{array}{l}\text { Conservatrice, } \\
\text { et autoritaire }\end{array}$ & Délibérative & Critique & Révolutionnaire \\
\hline Archétype & $\begin{array}{c}\text { L'étudiant qui } \\
\text { n'assiste pas à la } \\
\text { conférence et qui } \\
\text { se sert au buffet } \\
\text { destiné aux } \\
\text { convives }\end{array}$ & $\begin{array}{l}\text { Don de la } \\
\text { charité }\end{array}$ & $\begin{array}{l}\text { Organisation de } \\
\text { la charité }\end{array}$ & Activisme & $\begin{array}{l}\text { Citoyen } \\
\text { révolutionnaire }\end{array}$ \\
\hline
\end{tabular}

Tableau 2. Caractéristiques des citoyens selon la typologie de Westheimer et Kahne, adaptée par Lefrançois, Éthier et Demers (2009)

En dernier lieu, dans leur examen des typologies, Lefrançois, Éthier et Demers (2009, p.84) sousentendent l'existence théorique d'un dernier type de citoyen, soit le citoyen anomique/aliéné. Égoïste, ce dernier parasite les systèmes et s'en sert à son propre profit. Il suit ses propres règles ou les renie selon son intérêt personnel. Il n'agit pas au nom d'une idéologie ou d'un idéal: il fait ce qui lui semble nécessaire.

Nous proposons d'analyser deux cas afin d'identifier les recoupements entre la conception des droits et de la citoyenneté néolibérale et minimale et le traitement de l'exercice d'une agentivité citoyenne plus substantive par des élèves du secondaire.

\section{La fouille des élèves et le code vestimentaire: deux cas de figure du rapport au droit et de la formation citoyenne des élèves}

\subsection{La fouille des élèves à l'école}

La fouille des élèves dans les écoles québécoises a occupé une place importante de l'espace médiatique récemment. Le cas d'une élève soupçonnée de vendre de la drogue et fouillée à nue par le 
personnel de l'école secondaire de Neufchâtel (Lachance, 2015) a obligé le ministère de l'Éducation, de l'Enseignement supérieur et de la recherche à enquêter sur les situations de fouille d'élèves en raison des dilemmes éthiques et des questions légales que ces fouilles d'élèves soulevaient dans le public. En effet, cette pratique est répandue et dispose d'un encadrement légal plus souple que la fouille de citoyens adultes à l'extérieur de l'école: le droit actuel enjoint les écoles à procéder à des fouilles sans mandat si la sécurité immédiate des élèves est en jeu (R c. M. [1998] 3 R.C.S. 393). Les élèves devraient donc avoir une attente réduite en ce qui a trait au respect de leur vie privée lorsqu'ils sont à l'école (Bouchard, 2015).

La définition du motif légitime pour évaluer s'il existe une menace à la sécurité est floue lorsqu'appliquée dans les écoles (des dénonciations d'un pair, jugé crédible, par exemple, serait un motif légitime), par les directions d'établissement. De plus, les directions ainsi que les enseignants peuvent procéder à une fouille et peuvent le faire sans présence policière, bien que cette présence soit fortement recommandée (Bouchard, 2015). Enfin, le rapport du MEESR fait état de cas de contestation de l'absence de l'autorité parentale et parfois d'un avocat pendant la fouille. Un élève qui aurait demandé cet accompagnement se les seraient vus initialement refuser par la direction de l'école, qui face à son refus de coopérer à la fouille aurait finalement accepté que l'élève soit accompagné par un parent et ait accès à un avocat. Cet élève a refusé la fouille.

Certaines commissions scolaires et écoles possèdent une politique claire et des ententes avec un corps policier sur le sujet, alors que d'autres n'en ont pas ou dénoncent un manque de ressource donnant lieu à un résultat analogue à l'absence d'entente. Les constats de l'enquête sur le nombre en apparence important d'abus des autorités scolaires, le manque de formation de son personnel, le manque de ressource et l'absence de politique générale de coordinations des acteurs auraient comme conséquence de léser les droits des élèves. L'absence de coordination des acteurs favorise les dérapages de la part d'acteurs individuels.

Quels rapports au droit émergent de ces situations scolaires vécues par des élèves? En mobilisant la typologie d'Ewick et Silbey (1998) et à la lumière de l'information contenue dans le rapport d'enquête (Bouchard, 2015), le jugement de la cour suprême et du cadre de référence sur la présence policière dans les écoles, il est possible de conclure que le rapport au droit des élèves en question n'est pas nécessairement informé par une connaissance de leurs droits. Le seul cas d'élève qui semble avoir réussi à résister à l'autorité du droit mobilisé par la direction implique une compréhension du droit à une représentation légale. Dans ce cas, il est possible de postuler que la connaissance de certains éléments du droit, c'est-à-dire le fait de disposer de ressources, ait permis à l'élève d'agir avec le droit. Toutefois, pour les autres élèves, la formation à propos de leurs droits en matière de fouille consiste dans les faits à les placer devant le droit lorsqu'ils font l'objet d'une telle fouille.

Si elle traite principalement de l'action de l'État et des droits et responsabilité de l'école de protéger les élèves contre les armes et les drogues illicites, l'enquête du MEESR (Bouchard, 2015) reste, comme le jugement de la cour suprême, plutôt silencieuse sur les ressources mises à la disposition des élèves pour protéger leurs droits, ainsi que sur le caractère constitutionnel de certaines dispositions règlementaires des écoles. Les directions d'école expliquent-elles aux élèves quels sont ces droits, avant de procéder à une fouille? Les constats du rapport ne font état d'aucune situation où cela aurait été le cas.

Si les défenseurs de la fouille des élèves la présentent comme une stratégie de dissuasion justifiée pour faire face aux problématiques de drogues, d'intimidation et de violence dans les écoles, ses critiques se demandent plutôt comment éduquer un citoyen respectueux du droit d'autrui et du droit à la vie privée lorsque pour procéder à la fouille de citoyens mineurs, les critères légaux sont inférieurs à ceux qui sont mobilisés à l'extérieur de l'école. Dans tel cas, bien que le curriculum formel aborde les droits dans les perspectives sociohistorique et éthique, le curriculum caché actualisé dans l'exercice d'autorité et de soumission dans le cas des fouilles, par exemple, pourrait mener les élèves à considérer que le pouvoir des adultes et du droit les éloigne de toute possibilité d'agir avec le droit ou contre le droit («might makes right »). En effet, les cas recensés dans le Rapport du MEESR (Bouchard, 2015) 
portent à conclure que les élèves échouent à faire valoir leurs droits s'ils résistent seuls à l'autorité scolaire et doivent implorer la solidarité parentale et avoir recours à un avocat pour faire respecter leurs droits. La résistance emploie donc principalement la voie des tribunaux (où les parents agissent avec le droit) pour préciser l'application du droit de fouille des écoles. Or, dans ses décisions, la cour mobiliserait autant les responsabilités des institutions scolaires que des élèves (et de leurs parents) dans le maintien de "l'ordre et de la discipline » (Table provinciale de concertation sur la violence, les jeunes et le milieu scolaire, 2010, p. 9) pour justifier une attente réduite de respect des droits et libertés fondamentales des élèves. Par ailleurs, seuls les parents qui disposent des ressources financières pourraient s'engager dans une telle démarche. Il est possible de postuler que la remise en question de la fouille, la problématisation d'une attente réduite de respect de ses droits (qui relève toutes deux d'un rapport contre le droit) et de l'injustice économique qui réduit la possibilité d'agir avec le droit, soient difficilement possible pour les élèves. Cela réduit ainsi la possibilité pour eux de vivre cette situation comme citoyen orienté vers la justice, voire même de disposer de l'agentivité politique nécessaire pour agir de manière collective avec le droit que l'on associe à la citoyenneté participative.

\subsubsection{Analyse des cas liés à la fouille}

Quel citoyen peut être formé par l'expérience de la fouille à l'école? Les hypothèses que nous formulons ici ne sont que des hypothèses. À l'instar de Raby, nous reconnaissons l'impossibilité d'attribuer hors de tout doute l'émergence d'un type ou un autre de citoyenneté à la seule expérience scolaire, et certainement à la seule expérience d'un évènement unique (bien que marquant). Toutefois, l'exercice a ceci d'intéressant: il souligne le potentiel politique du rapport que les élèves peuvent entretenir au droit dans le cadre de leur vie scolaire quotidienne et ainsi, le rôle que jouent les acteurs de l'école dans la socialisation citoyenne.

L'expérience de l'élève qui a refusé la fouille et obtenu la présence d'un parent, ainsi que l'accès à une représentation légale, est fort différente de celle des autres élèves, qui ont subi la fouille contestée par la suite devant les tribunaux par les parents, ou encore qui ont subi la fouille sans que la contestation n'inclue le recours aux tribunaux. Dans le premier cas, en mobilisant la typologie de Westheimer et Kahne (2004), il est possible de postuler que l'élève ayant agi avec le droit pour résister à son application arbitraire et donc ayant vécu l'expérience d'une réussite de sa participation active au sein des institutions publiques pour résoudre un problème puisse développer une certaine confiance en ces institutions et en sa capacité d'y agir, associée à la citoyenneté participative.

Dans le cas des autres élèves, ceux qui n'ont pas contesté la fouille et qui ont été placés devant le droit comme institution immuable et à laquelle ils doivent obéissance auront peut-être choisi l'obéissance au droit afin d'éviter le conflit et parce qu'ils croient à son objectivité et à sa légitimité. Dans ce cas, il serait possible d'extrapoler que cette expérience alimente une citoyenneté personnellement responsable, ancrée dans l'obéissance aux institutions transcendantes avec lesquelles il entretient un rapport minimaliste. Il est toutefois également possible d'avancer que, pour certains de ces élèves, l'absence de contestation est moins le fruit d'une obéissance que d'une résignation à leur impuissance individuelle, leur absence de pouvoir pour agir contre le droit. La typologie de Westheimer et Kahne nous informe toutefois peu sur les pratiques sociales du citoyen qui peut être formé par une telle expérience, mais les ajouts conceptuels de d'Éthier, Lefrançois et Demers (2009) nous permettent d'avancer qu'il pourrait s'agir d'une forme de renoncement de s'engager auprès des institutions publiques, c'est-à-dire d'une forme d'aliénation.

Dans le cas des élèves dont les parents auront eu recours aux tribunaux pour défendre leurs intérêts, il est difficile de savoir si cette action avec le droit a été réalisée dans l'intention de créer une jurisprudence plus respectueuse des droits des élèves, c'est-à-dire de négocier avec le droit dans un intérêt public, collectif ou simplement dans un intérêt particulier. Cette expérience vécue par les élèves peut leur offrir une meilleure compréhension des institutions publiques s'ils participent activement au processus juridique, dans tel cas, pourrait alimenter la formation d'une citoyenneté plutôt participative. Si toutefois les élèves ne sont pas impliqués dans le processus, leur expérience sera limitée à avoir vécu l'imposition du droit par les autorités scolaires et peut-être au partage d'informations au sujet du 
processus juridique par les parents. Il pourrait en découler une citoyenneté soit participative, dans le cas où l'élève s'approprie le processus de contestation, soit personnellement responsable, si l'élève se résigne à accepter le pouvoir des institutions publiques et un devoir de s'y conformer.

\subsection{Le code vestimentaire}

Si les cas de fouille des élèves à l'école semblent vécus de façon atomisée, plaçant l'élève seul devant l'adulte en autorité, les horizons d'action «citoyenne» avec et contre le droit (les règles scolaires) des élèves semblent plus importants et porteurs d'une citoyenneté participative ou orientée vers la justice lorsque la problématique est vécue collectivement. À ce titre, les codes vestimentaires qui visent à réguler plus en particulier le corps des élèves s'identifiant au sexe féminin présentent des cas de figure intéressants.

En 2016, des élèves de l'École secondaire Robert-Gravel, qui venait d'adopter dans son code de vestimentaire l'obligation pour les filles de porter le soutien-gorge, se sont mobilisés à l'encontre d'une règle qu'elles et ils ont considérée sexiste et discriminatoire pour les élèves s'identifiant au sexe féminin (Nadeau, 2016). Dans leur campagne de dénonciation, ces élèves ont remis en question la règle et tenté de la faire changer en invoquant l'injustice qu'elle représentait pour les élèves s'identifiant au sexe féminin. Toutefois, confrontés à une réponse insatisfaisante des acteurs du pouvoir scolaire, ces élèves ont dénoncé le nouveau code publiquement, fait grève et, dans une action forte contre le droit (que représentait la nouvelle règle), ont suspendu leur soutien-gorge au cadenas de leur casier d'école, alors que des garçons ont porté des soutien-gorge par-dessus leurs vêtements. Face à cette résistance collective et publique, la direction de l'école a alors entamé des discussions avec les élèves mobilisés pour entendre leurs revendications et trouver une solution. Citant l'importance de l'éducation à la démocratie, l'école s'est pliée aux demandes des élèves et les a félicités pour leur prise de parole.

\subsubsection{Analyse du cas lié au code vestimentaire}

Le rapport au droit manifesté par les élèves de l'École Robert-Gravel se distingue de celui manifesté dans les cas de fouilles sur deux plans. En premier lieu, il n'implique pas par extension le code criminel et il est mobilisé par un groupe, c'est-à-dire collectivement. Confrontés comme groupe à une règles qui leur est parue injuste, les élèves en ont problématisé les origines, pour arriver à un constat d'injustice et de l'exercice d'un contrôle discriminatoire envers un groupe social. Dans ce cas, la règle allait bien au-delà du vêtement, pour réguler l'apparence physique (ce qui pourrait être tout aussi questionnable), mais bien le corps féminin, le soutien gorge étant porté sous le vêtement de façon à donner une forme contrainte aux seins. L'analyse qu'ont fait les élèves du nouveau règlement reposait d'abord sur la mobilisation de droits et libertés fondamentales (l'égalité devant la loi) et pourrait ainsi être associée à un agir avec le droit. Si les élèves avaient eu gain de cause avec le droit, il aurait été possible de reconnaître dans leur action à l'intérieur des institutions et structures de pouvoir existantes une citoyenneté participative. Toutefois, le refus des autorités scolaires de retirer le nouveau règlement a mené à agir contre le droit (la règle scolaire et le pouvoir légal du Conseil d'établissement de la fixer) dans la défense de droits fondamentaux. S'agit-il toujours ainsi d'une action que l'on pourrait associer à un rapport avec le droit, ou contre le droit puisqu'il relève de la désobéissance comme acte de résistance? En d'autres mots, l'expérience de résistance et de contestation vécue par les élèves (ainsi que sa réussite, en termes d'effets) peut-elle alimenter la formation d'une citoyenneté participative, qui agit à l'intérieur et par les institutions publiques, ou plutôt celle d'une citoyenneté orientée vers la justice et prête à remettre en question, y compris par l'action, les structures sous-jacentes au pouvoir de ces institutions? Nous posons que les deux résultats sont possibles.

\section{Discussion}

L'analyse des deux cas de figure présentée plus haut nous mène à constater que la typologie de rapport au droit de Ewick et Silbey (1998) peut effectivement être féconde, bien que perfectible et incomplète, pour analyser l'interaction entre les élèves et les codes normatifs de l'institution scolaire, chargée de leur formation citoyenne. Dans son extension analogique, le rapport au droit permet de 
qualifier la posture des élèves face à l'institution publique (le droit) qui organise les rapports sociaux dans leur quotidien et peut être en ce sens d'une grande utilité pour ceux qui souhaitent le façonner par l'éducation ou encore comprendre comment il se développe au sein d'une institution (Silbey, 2005). Conjuguée à la typologie des finalités de l'éducation à la citoyenneté, le rapport au droit permet d'envisager cette posture dans un contexte politique plus large, soit celui de la polis, au sein de laquelle les citoyens agissent de façon individuellement responsable face aux institutions publiques, y participent activement ou les remettent en question, les problématisent et en dénoncent les asymétries dans une perspective de justice sociale.

Toutefois, cette typologie est plus difficile à actualiser pour analyser des manifestations d'une remise en question réfléchie du droit et des injustices structurales qui peuvent y être inhérentes. Dans son application comme cadre d'analyse, la typologie ne semble pas inclure ce rapport critique et contestataire. Le rapport contre le droit, dans la définition conceptuelle qu'offrent Ewick et Silbey pourrait s'y extensionner, mais ne semble pas inclure de contestation de l'institution publique établie hors d'une conception criminelle ou parasitaire. En ce qui concerne la typologie de Westheimer et Kahne (2004), elle présente certaines limites quant aux distinctions dans le rapport aux institutions publiques comme le droit entre le citoyen participatif et le citoyen orienté vers la justice. C'est notamment le cas des expériences qui placent le droit (la règle et l'autorité qui assure son respect) en confrontation avec les droits et libertés fondamentales. Comme l'École tend à être présentée dans les jugements des tribunaux comme une zone de suspension ou de réduction de ces droits et libertés (qui existeraient «sous réserve, toujours, de la discipline scolaire normale» selon la Cour d'appel du Québec, dans un extrait cité par Bouchard [2015]) la distinction nous parait importante et nous pousse à questionner dans quelle mesure les droits et libertés sont considérés par les acteurs et usagers de l'École comme faisant partie du droit et constituant ainsi des normes incontournables dans l'organisation des rapports sociaux et la capacitation d'une citoyenneté orientée vers la justice.

Les recoupements entre les deux typologies nous apparaissent à la fois importants et complémentaires. Bien qu'elles diffèrent dans leur traitement de l'intention des acteurs sociaux, toutes deux sont orientées vers le sens que donnent les acteurs sociaux aux structures et institutions qui façonnent leur quotidien. Dans le cas du rapport au droit comme dans celui de l'éducation à la citoyenneté, les auteurs reconnaissent que l'expérience d'une situation plaçant ces deux institutions en jeu est essentielle pour que les acteurs puissent en construire le sens. Ce constat nous apparait fondamental dans une perspective éducative: si les élèves peuvent lire au sujet du droit et de la citoyenneté dans les manuels scolaires, en mémoriser les théories et définitions, l'expérience qu'ils font de ces institutions dans leur quotidien exerce néanmoins un effet plus important sur le sens qu'ils en construisent que n'en a un cours formel. À ce titre, les codes de conduites et règles scolaires constituent un vecteur d'expériences éducatives non négligeable sur le plan de la formation citoyenne et doivent occuper une place plus importante dans l'analyse du rôle de l'école dans la socialisation politique des jeunes.

\section{Références bibliographiques}

Adelman, M. et Yalda, C. (2000). Seen but not heard. The legal lives of young people. PoLAR: Political and Legal Anthropology Review. 23(2), 37-58. doi :10.1525/pol.2000.23.2.37

APPLE, M. (2004). Ideology and the Curriculum. New York : Routledge.

AUdigier, F. (2007). L'éducation à la citoyenneté dans ses contradictions. Revue internationale d'éducation de Sèvres, (44), 25-34. doi:10.4000/ries.125

BernsteIN, B. (1977). Class, Codes and Control. Volume III. London : Routledge et Kegan.

BIBLIOTHEQUE DU PARLEMENT (2012). Résumé législatif du projet de loi C-10: 9 Modifications à la Loi sur le système de justice pénale pour les adolescents [c-10, partie 4, art. 167 à 204 - ancien projet de loi C-4]. Repéré en ligne le 9 décembre $2016 \quad$ http://www.lop.parl.gc.ca/About/Parliament/LegislativeSummaries/bills 1s.asp?1s=c10$\underline{09 \& \mathrm{Parl}=41 \& \text { Ses }=1 \& \text { Language }=\mathrm{F} \# \mathrm{ftn} 2}$

BOUCHARD, F. (2015). Rapport d'enquête sur la fouille d'élèves. Québec: Gouvernement du Québec. 
Bourdieu, P. (1972). Esquisse d'une théorie de la pratique. Paris : Droz. Repéré à https://www.cairn.info/esquisse-dune-theorie-de-la-pratique--9782600041553.htm

Bowles, S. et GinTIS, H. (2011). Schooling in Capitalist America: Educational Reform and the Contradictions of Economic Life. New York, NY : Basic Books.

Brisman, A. (2012) Legal Consciousness Among Youth at the Red Hook Community Justice Center. Thèse de doctorat, Emory University.

CARR P.J., NAPOLITANO L., et KEATING J. (2007). We never call the cops and here is why: A qualitative examination of legal cynicism in three Philadelphia neighborhoods. Criminology. 45, 445-480.

Chriss, J. J. (2007). Social control: an introduction. Cambridge, UK ; Malden, MA: Polity.

COUR SUPREME DU CANADA. R. c. M. (M.R.), no. 26042 RCS § 3 393-436 (1998). Repéré à https://scccsc.lexum.com/scc-csc/scc-csc/fr/item/1666/index.do

CRICK, B. (2007). Citizenship: The political and the democratic. British Journal of Educational Studies, 55(3), 235-248. doi:10.1111/j.1467-8527.2007.00377.x

DEMERS, S. (2011). Relations entre le cadre normatif et les dimensions téléologique, épistémologique et praxéologique des pratiques d'enseignants d'histoire et éducation à la citoyenneté: étude multicas (Thèse de doctorat, Université du Québec à Montréal, Montréal, Québec). http://www.collectionscanada.gc.ca/obj/thesescanada/vol2/QMUQ/TC-QMUQ-5079.pdf

DEVINE, D, 2002. Children's citizenship and the structuring of adult-child relations in the primary school, Childhood, 9(3), 303-320.

DREBEEN. R. (1968). On what is learned in schools. Reading, Mass.: Addison Wesley.

DuRKheIM, E. (1934/2008). L'éducation morale. Paris : Librairie Félix Alcan. Mis en ligne par Tremblay, J.M. Les Classiques des sciences sociales. Université du Québec à Chicoutimi.

Eid, P., MAgloire, J. et TuRenNe, M. (2011). Profilage racial et discrimination systémique des jeunes racisés: rapport de la consultation sur le profilage racial et ses conséquences. Québec, Qué. : Commission des droits de la personne et des droits de la jeunesse. Repéré à http://public.eblib.com/choice/publicfullrecord.aspx?p=3275047

ÉTHIER, M.-A. (2001). Où va l'éducation à la citoyenneté? Traces, 39(3), 17-28.

ÉTHIER, M.-A. (2003). Du statut d'un cadre conceptuel dans une recherche qualitative. Recherches qualitatives, 23, 3-13.

EwICK, P. et SiLBEY, S. (1998). The common place of law: stories from everyday life. Chicago : University of Chicago Press.

Feldman, D. (2007). Citizenship Education: Current perspectives from teachers in three states. Educational Research Quarterly, 30 (4), 3-15.

FouCAULT, M. (1977). Surveiller et punir: naissance de la prison (Réimpression). [Paris] : Gallimard.

FriedenberG, E. Z. (1969). Current Patterns of Generational Conflict. Journal of Social Issues, 25(2), 21-38. doi:10.1111/j.1540-4560.1969.tb00593.x

GirouX, H. et A. PenNA. (1979). Social education in the classroom: the dynamics of the hidden curriculum. Theory and Research in Social Education, 7(1), 21-42.

GiROUX, H. A. (1981). Hegemony, resistance, and the paradox of educational reform. Interchange, 12(2-3), 326. doi:10.1007/BF01192105

GiRouX, H. A. (2000). At war against the young: Corporate culture, schooling and politics of «zero tolerance. » Against the Current, 86, 17-21.

GIROUX, H. A. (2003). The abandoned generation: democracy beyond the culture of fear (1st ed). New York : Palgrave Macmillan.

Giroux, H. A. (2009). Hard Lessons: Neoliberalism, Education, and the Politics of Disposability. Policy Futures in Education, 7(5), 570-573. doi:10.2304/pfie.2009.7.5.570

Giroux, H. A. (2016, 19 octobre). Henry A. Giroux | The United States' War on Youth: From Schools to Debtors' Prisons. Truthout. Repéré 9 décembre 2016, à http://www.truth-out.org/news/item/38044-america-s-war-on-youthfrom-schools-to-debtors-prisons

Hagan, J., ShedD, C. et PAyne, M. R. (2005). Race, Ethnicity, and Youth Perceptions of Criminal Injustice. American Sociological Review, 70(3), 381-407. doi:10.1177/000312240507000302 
HIRSHFIELD, P. (2008). Preparing for prison?: The criminalization of school discipline in the USA. Theoretical Criminology, 12(1), 79-101.

JACKSON, P. (1968). Life in Classrooms. New York : Holt, Rinehart \& Winston.

JaKubowsKa, I. (1991). Droit et justice chez les enfants et les adolescents, Droit et Société, 19, p. 287-295.

KAHNE, J. et WeStheimer, J. (2006). The limits of political efficacy: Educating citizens for a democratic society. PS: Political Science \& Politics, 39(2), 289-296.

KoHlBerg, L. (1976). Moral Stages and Moralization : The Cognitive-developmental Approach . Dans T. LIKONA (dir.), Moral Development and Behavior: Theory, Research, and Social Issues (p. 31-53). New York, Holt, Rinehart and Winston.

LACHANCE, N. (2015, février). «Je me sentais violée». Le Journal de Québec. Repéré 1er mai 2017, à http://www.journaldemontreal.com/2015/02/16/je-me-sentais-violee

LEFRANÇOIS, D. (2006a). La formation civique devant la diversité des modèles normatifs de la citoyenneté. Dans L. CHEVALIER (dir.), Le politique et ses normes. Les débats contemporains en philosophie politique (p. 77-91). Rennes : Presses Universitaires de Rennes.

LEFRANÇOIS, D. (2006b). Défense d'un modèle délibératif de la citoyenneté et analyse de ses implications normatives en matière de formation civique: perspectives philosophiques sur l'éducation à la citoyenneté dans le contexte de la réforme éducative québécoise (Thèse de doctorat, Université du Québec à Trois-Rivières, Trois-rivières, Québec). Repéré à http://depot-e.uqtr.ca/1284/1/030001625.pdf

Lefrançois, D., Éthier, M.-A. et Demers, S. (2009). Justice sociale et réforme scolaire au Québec: le cas du programme d' «Histoire et éducation à la citoyenneté». Éthique publique. Revue internationale d'éthique sociétale et gouvernementale, 11(1), 72-85. doi:10.4000/ethiquepublique.1327

LEMAY, V. (2000). Évaluation scolaire et justice sociale: droit, éducation et société. Montréal, Québec : Éditions du Renouveau pédagogique.

LESSARD, J.-F. (2008). La question du politique dans la modernité. Comprendre le malaise contemporain. Montréal, Québec : Liber.

Merry, S. E. (1985). Concepts of law and justice among working-class Americans: Ideology as culture. Legal Studies Forum, 9, 59.

MinisTERE DE L'ÉDUCATION. (2004). Programme de formation de l'école québécoise. Enseignement secondaire - 1er cycle. Québec, Québec : Gouvernement du Québec.

MorriLl, C., TYSON, K., Edelman, L. B. et Arum, R. (2010). Legal Mobilization in Schools: The Paradox of Rights and Race Among Youth: Legal Mobilization in Schools. Law \& Society Review, 44(3-4), 651-694. doi:10.1111/j.1540$\underline{5893.2010 .00419 . x}$

NADEAU, J. (2016). Mobilisation colorée contre le soutien-gorge obligatoire. Le Devoir. Repéré à http://www.ledevoir.com/societe/education/472022/ecole-robert-gravel-mobilisation-coloree-contre-le-soutien-gorgeobligatoire

O’NeILl, J. (1973). Embodiment and Child development: a phenomenological approach. Dans H.P. Dreitzel (dir.), Childhood and Socialization (pp. 65-81). New York : Macmillan.

PAGE, M. (2001). L'éducation à la citoyenneté devant la diversité des conceptions de la citoyenneté. Dans M. PAGE, F. OUELLET et L. CORTESÃO (dir.), L'éducation à la citoyenneté (p. 41-54). Sherbrooke : Éditions du CRP.

Pelisse, J. (2005). A-t-on conscience du droit? Autour des Legal Consciousness Studies. Genèses, 59(2), 114-130.

PrINT, M. (2007). Citizenship Education and Youth Participation in Democracy. British Journal of Educational Studies, 55(3), 325-345. doi:10.1111/j.1467-8527.2007.00382.x

Prost, A. (1997). Éducation, société et politiques: une histoire de l'enseignement en France de 1945 à nos jours (Nouvelle édition augmentée). Paris : Éditions du Seuil.

RABY, R. (2005). Polite, well-dressed and on time: Secondary school conduct codes and the production of docile citizens. The Canadian Review of Sociology, 42(1), 71-91. doi:10.1111/j.1755-618X.2005.tb00791.x

RABY, R. (2008). Frustrated, Resigned, Outspoken: Students' Engagement with School Rules and Some Implications for Participatory Citizenship. The International Journal of Children's Rights, 16(1), 77-98. doi:10.1163/092755608X267148

SARAT, A. (1990). The law is all over: power, resistance and the legal consciousness of the welfare poor. Yale Journal of Law \& the Humanities, 2, 343-379. 
Sarat, A. et Silbey, S. (1988). The Pull of the Policy Audience. Law \& Policy, 10(2-3), 97-166. doi:10.1111/j.14679930.1988.tb00007.x

SCOTT-BAUMANN, A. (2003). Citizenship and postmodernity. Intercultural Education, 14(4), 355-366. doi:10.1080/1467598032000139813

SiLbeY, S. S. (2005). After Legal Consciousness. Annual Review of Law and Social Science, 1(1), 323-368. doi:10.1146/annurev.lawsocsci.1.041604.115938

Silbey, S. S. et BitTner, E. (1982). The Availability of Law. Law \& Policy, 4(4), 399-434. doi:10.1111/j.14679930.1982.tb00284.x

StATistiQues CANADA. (2016). La criminalité chez les jeunes au Canada, 2014. Le Quotidien, Composante du produit no. 11-001-X au catalogue de Statistique Canada. Repéré à http://www.statcan.gc.ca/dailyquotidien/160217/dq160217b-fra.pdf

STEVens, H. R. (2013). Rules, Laws and Conceptions of Justice in Middle School: An Exploratory Study of Children's Legal Consciousness (Thèse de doctorat, George Mason University, Fairfax, VA). Repéré à http://hdl.handle.net/1920/8351

SURPRENANT, H. (2003). L'éducation à la citoyenneté comme forme de socialisation juridique: pour une prise de conscience de ses droits et devoirs fondamentaux dans une perspective contemporaine (Mémoire de maîtrise, Université de Montréal, Montréal, $\quad$ Québec). https://papyrus.bib.umontreal.ca/xmlui/bitstream/handle/1866/2767/11449335.PDF

TABLE PROVINCIALE DE CONCERTATION SUR LA VIOLENCE, LES JEUNES ET LE MILIEU SCOLAIRE (2010). Présence policière dans les établissements d'enseignement. Cadre de référence. Récupéré en ligne le 16 décembre 2016 http://www.sq.gouv.qc.ca/parent-et-enseignants/cadre-de-reference/cadre-de-reference.pdf

TAPP, J. L. et KoHlBerg, L. (1971). Developing Senses of Law and Legal Justice. Journal of Social Issues, 27 (2), p. 6591

Westheimer, J. et Kahne, J. (2004). What Kind of Citizen? The Politics of Educating for Democracy. American Educational Research Journal, 41(2), 237-269. doi:10.3102/00028312041002237

Weinstock, D. (2000). La citoyenneté en mutation. Dans Y. Boisvert, J. HAMEL et M. Molgat (dir.), Vivre la citoyenneté. Identité, appartenance et participation (p. 15-26). Montréal : Liber.

YounG, M. (1976). Knowledge and Control. London : Collier MacMillan. 